\title{
ZUR HERKUNFT DER POLONAISE BWV ANHANG 130
}

\author{
Karl-Heinz V i e r t e l (Leipzig)
}

Im Kritischen Bericht des Klavierbüchleins für Anna Magdalena Bach1 beklagt der Herausgeber Georg von Dadelsen, daß sich die "Autoren der vielen anonymen Sätze... nur in beschränktem Umfange ermitteln ließen«.2

Verschiedentlich konnten nun in den letzten Jahren einige Johann Sebastian Bach zugeschriebene oder bereits als zweifelhaft erkannte Werke bezüglich ihrer Herkunft identifiziert werden. Als markante Beispiele seien hier lediglich zwei angeführt: Cornelius Heinrich Dretzel ist der tatsächliche Autor von Präludium und Fuge für Cembalo BWV 897 - Isolde Ahlgrimm hat darüber im Bach-Jahrbuch 1969 ausführlich berichtet ${ }^{3}$ und es gelang dem polnischen Musikwissenschaftler Karol Hławiczka die Provenienzen zweier Bachscher Polonaisen aus alt-polnischen Quellen uiberzeugend $\mathrm{zu}$ erhellen. ${ }^{4}$

Einem glücklichen Zufall ist es nun $\mathrm{zu}$ verdanken, daß bezüglich der Polonaise Nr. 28 (G-Dur), BWV Anhang 130 aus dem Klavierbüchlein für Ana Magdalena Bach der Komponist entdeckt werden konnte. Es ist mit hoher Sicherheit Johann Adolf Hasse, der von 1733 bis 1763 in Dresden als Königlich-Polnischer und Kurfürstlich-Sächsischer Kapellmeister (seit 1750 als Oberkapellmeister) gewirkt hat. ${ }^{5}$

In der Staatsbibliothek Preußischer Kulturbesitz Berlin, Musikabteilung, wird eine Handschrift verwahrt (Signatur: BB Mus. ms. 9640), die unter der Autorschaft Johann Adolf Hasses "Sonate e Sinfonie per il Cem-

1 Johann Sebastian Bach, Neue Ausgabe sämtlicher Werke, Serie V, Band 4, Deutscher Verlag für Musik, Leipzig, 1957.

${ }^{2}$ Kritischer Bericht, p. 72.

${ }^{3}$ Ahlgrimm I., Cornelius Heinrich Dretzel, der Autor des J. S. Bach zugeschriebenen Klavierwerks BWV 897. in: Bach-Jahrbuch 1969, p. 67-77.

${ }^{4}$ Hławiczka K., Die Herkunft der Polonaise-Melodie der Ouvertüre $h$-Moll (BWV 1067), in: Bach-Jahrbuch 1966, p. 99-101.

${ }^{5}$ Dieser Sachverhalt stellte sich bei Vorbereitungsarbeiten für die Edition Hasseschen Cembalo-Sonaten innerhalb der Reihe "Musica alla corte sassone-polaccau heraus, einer Gemeinschafts-Edition zwischen VEB DVfM Leipzig und PWM Kraków. Mit dem Erscheinen der ersten beiden Bände ist in absehbarer Zeit zu rechnen. 
balo« überliefert, so die Beschriftung auf dem äußeren Buchdeckel. Es ist die Handschrift eines unbekannten, resp. bis jetzt noch nicht ermittelten Kopisten, welche in einen grauen Pappeinband mit blauem Rücken gebunden ist; Format der Blätter 29,4 $\mathrm{cm} \times 21,0$ quer. Erhaltungszustand des Bandes: ausgezeichnet.

Bei den Sinfonien, die in unserem Zusammenhang ohne Bedeutung sind, handelt es sich um Cembalo-Versionen (wir würden heute sagen: Klavierauszugfassungen) von folgenden Hasseschen Werken:

1. dem Oratorium I PELLEGRINI AL SEPOLCRO (Mennicke Nr. 8) ${ }^{6}$

2. der Oper ALCIDE AL BIVIO

(Mennicke Nr. 51)

3. der Oper

(= Die Wahl des Herakles)

4. der Oper

EZIO

5. der Oper

LEUCIPPO

6. dem Oratorium

SOLIMANO

(Mennicke Nr. 45)

(Mennicke Nr. 77)

(Mennicke Nr. 70)

ST. ELENA AL CALVARIO

(Mennicke Nr. 9)

Diesen sechs Sinfonien ( = Opern-Ouvertüren) sind vorangebunden sieben Cembalo-Sonaten Hasses, die im Innern jeweils (bis auf eine Ausnahme, die Sonate Nr. 6) mit einem gesonderten Titelblatt versehen sind, das die Formulierung enthält "Sonata per il Cembalo, del Sigr. Giov. Adolfo Haße detto il Sassoneu (Sonaten 1, 3, 4, 5, 7). Bei zwei Sonaten heißt es "Sonata per Cembalo, del Sigr. Giov. Adolfo Haße detto il Saßone" (Titelblatt Nr. 2 und U̇berschrift Nr. 6).

Fünf Sonaten dieses Berliner Bandes enthält auch eine Handschrift der Sächsischen Landesbibliothek Dresden, Signatur: Mus. $2477-\mathrm{T}-3$. Beide Manuskripte, dâs Dresdener und das Berliner, weisen einen hohen tbereinstimmungsgrad des Notentextes auf.7 Lediglich zwei der Berliner Sonaten (Nr. 1 und 2) sind nicht im Dresdener Ms. enthalten.

Bei Nr. 2 - der für uns Relevanten - fällt zunächst ins Auge, daß sie hinsichtlich der Satzfolge umfangreicher ausfällt, als bei den meisten Cembalo-Sonaten Hasses üblich. Dem einleitenden Allegro-Satz in C-dur (in sich geteilt durch Wiederholungen) folgt ein Andante im $3 / 8$ takt, dem sich ein Menuett mit Trio anschließt. Den Abschluß bilden zwei Polonaisen, der Orthographie der Zeit entsprechend als "Polonoise $1^{\circ}$ und $2^{\circ}$ * bezeichnet.

${ }^{6}$ Mennicke C., Hasse und die Brüder Graun als Symphoniker, Breitkopf \& Härtel, Leipzig, 1906. Darin befindet sich ein Them. Verzeichnis der Opern und Oratorien Hasses, p. 500-525, dessen entsprechende Nummern hier zur Orientierung mitgeteilt seien.

7 Hoffmann-Erbrecht L., Deutsche und italienische Klaviermusik zur Bachzeit. Studien zur Thematik und Themenverarbeitung in der Zeit von 1720-1760. Jenaer Beiträge zur Musikforschung, herausgegeben von Heinrich Besseler, VEB Breitkopf \& Härtel Musikverlag Leipzig, 1954, gibt diese in Berlin fehlende Sonate auf p. 140 (Incipit Nr. 9) irrtümlich als im Berliner Ms. befindlich an. Doch ist diese Sonate dort nicht enthalten. Dafür fehlen auf p. 141 (Incipit $13 \mathrm{u}$. 16) die Hinweise auf Quelle BB Mus. ms. 9640. Und zwar bei $\mathrm{Nr} .13=\mathrm{Nr}$. 1 und $\mathrm{Nr}$. $16=\mathrm{Nr}$. 2. Nähere Angaben siehe im Vorwort und Lesartenverzeichnis der unter Anm. 5 angeführten Hasseschen Cembalo-Sonaten (Dedikations-Sonaten für Maria Josepha). 
Polonaise Nr. 2 nun, obzwar bei Hasse in F-Dur stehend, ist identisch mit der im Notenbüchlein für Anna Magdalena Bach befindlichen Polonaise BWV Anhang 130, Nr. 28, G-Dur.
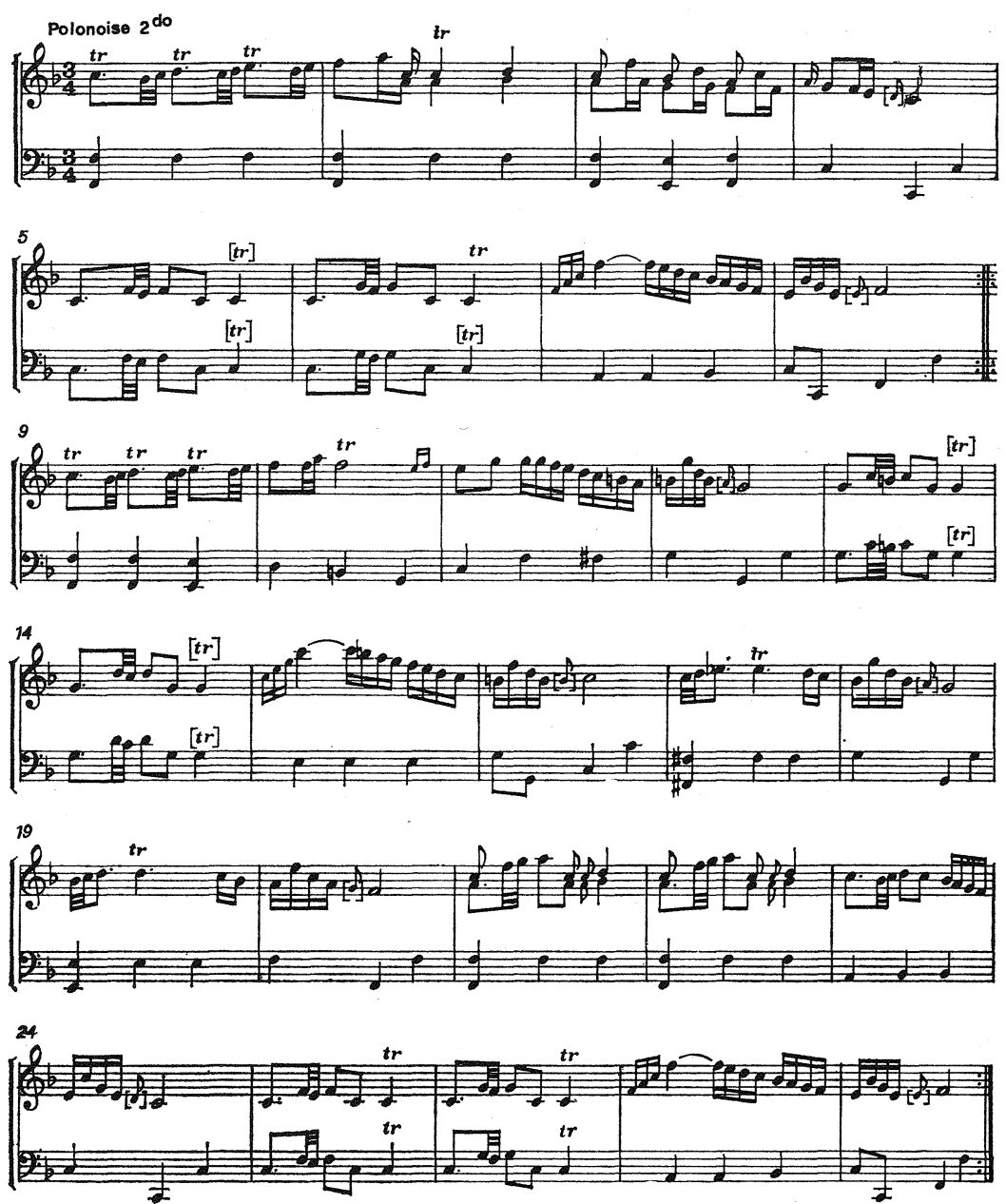

Beide Polonaisen weisen, wie aus dem Notenbeispiel ersichtlich, eindeutig den gleichen Melodieverlauf auf. Geringfügige Abweichungen wie in Takt 10 und 11 fallen nicht ins Gewicht, sie finden sich bei Kopistenabschriften im 18. Jahrhundert in vielfältiger Weise.

Ebenfalls nicht ins Gewicht fallen dürften die kleinen rhythmischen Verschiebungen Bachs zu Hasse, etwa in Takt 1 und der zur Dreistimmigkeit aufgefüllte Beginn Bachs Hasse gegenüber. Keinen Unterschied jedoch gibt es hinsichtlich der Taktzahl, sie beträgt übereinstimmend für den ersten Teil 8 Takte und den zweiten 20, insgesamt also 28 Takte. 
Besonders wichtig im Sinne der Polonaisen-Intonation sind bei Bach und Hasse übereinstimmend die von oben herabsinkenden Schlußwendungen in Takt 8 und 28. Sie entsprächen allerdings dem Polonaisen-Idealfall bekanntermaßen noch zwingender, wäre der Vorhalt von oben angesetzt. Dies ist jedoch der Fall in Hasses "Polonoise $1^{\circ} \|$. Es darf in diesem Zusammenhange durchaus vermutet werden, Hasse habe hier bewußt die beiden Polonaisen gerade in den Schlußwendungen voneinander abgesetzt. Hier das Beispiel für Takt 8 und 26 aus der ersten Polonaise:

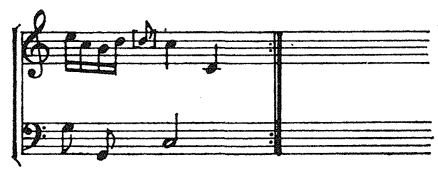

Es erhebt sich nun die Frage, ob es sich bei der mit Bach übereinstimmenden Polonaise um eine originale Komposition des Dresdener Hofkapellmeisters handelt, oder nur um eine U̇bernahme, resp. Kontrafaktur aus polnischer Folklore.

Zwei vorzügliche Kenner der polnischen Musik-Folklore, Zofia Stęszewska und Karol Hławiczka, konnten auf persönliches Befragen keine adäquaten Melodiemodelle einer Original-Polonaise mitteilen. ${ }^{8}$ Daher ist die Annahme sehr wohl berechtigt, es handele sich um eine Original-Komposition Hasses, zumal es Konkordanzen für Hasses Cembalo-Sonaten 1 und 2 des Berliner Ms. gibt. Für die hier vorrangig interessierende Sonate Nr. 2 ist es: British Museum London, Add. Ms. $32075^{9}$ (die einzusehen und mit der Berliner Handschrift zu vergleichen mir allerdings widriger Umstände wegen bis jetzt leider nicht möglich war) und für die Nr. 1: Sammlungen der Gesellschaft der Musikfreunde, Wien, VII 14, 524 III Nr. 1.10

Ein Autograph der Hasseschen Cembalo-Sonaten existiert leider nicht mehr. U̇berhaupt muß gesagt werden, daß wir von Hasse, im Verhältnis zu seinem großen Gesamtwerk, relativ wenig originale Handschriften besitzen - abgesehen von einigen Opernpartituren in italienischen Bibliotheken, insbesondere in der Biblioteca del Conservatorio di Musica "Giuseppe Verdi« Milano, Fondo Proprio - Musica teatrale, wo der musikalische Nachlass Hasses und seiner Frau Faustina Bordoni befindlich ist. Die

8 Siehe dazu Stęszewska Z., Polonica im Schaffen deutscher Komponisten des 16.-18. Jahrhunderts, und Hławiczka K., Einflüsse polnischer Musik in den Werken von J. S. Bach, beides in: Materiały do cyklu odczytów zorganizowanego przez Filharmonie Pomorska, Bydgoskie Towarzystwo Naukowe przy wspołudziale Związku Kompozytorów NRD - z okazji Bydgoskiego Festiwalu Muzycznego, 1974. Ferner Hławiczka K., Grundriss einer Geschichte der Polonaise bis zum Anfang des 19. Jahrhunderts, in: Svensk Tidskrift för musikforskning, Argang 50, Stockholm 1968, p. 51-124. Frau Dr. Stęszewska von der Polnischen Akademie der Wissenschaften Warszawa und Herrn Dr. Hlawiczka (Cieszyn) sei an dieser Stelle für ihre bereitwilligen Auskünfte herzlich gedankt.

- Siehe: Hoffmann-Erbrecht, a. a.O., p. 141, Incipit 16. Der 6. Ton der Oberstimme des Incipits lautet nach dem Berliner Ms. "d» (und nicht »e»).

10 Siehe ebenda, p. 141, Incipit 13. 
Gründe für diesen Sachverhalt habe ich an anderer Stelle dargelegt, sie bedürfen also an dieser Stelle keiner Erörterung. ${ }^{11}$

Hasse war nicht nur die musikalisch überragende Erscheinung im 18. Jahrhundert auf dem Gebiete der Opera seria (er hat ïbrigens auch bezaubernde Intermezzi geschrieben),12 dessen Werke $u$. a. in Neapel, Venedig, Dresden, London, Wien, Kopenhagen, St. Petersburg, Warschau und Moskau gespielt worden sind, sondern Hasse komponierte auch eine respektable Anzahl von Instrumental-Werken, die leider auch heute noch weithin unbekannt sind. Abschriften seiner Cembalo-Sonaten sind in vielen europäischen Bibliotheken nachweisbar, $u$. a. in Lund, Brüssel, London, Wien, Dresden, Cambridge, Berlin, Mailand, Leipzig, Bologna und Venedig (soweit der heutige Ermittlungsstand), müssen also seinerzeit verbreiteter gewesen sein, als wir heute anzunehmen geneigt sind.13 Auch in den Breitkopfischen Manuskripten Katalogen sind einige im Verlagsangebot nachweisbar, von 1763 sogar mit Incipits.

Wann und wo könnte nun Johann Sebastian Bach mit Hasses Polonaise bekannt geworden sein?

Georg von Dadelsen setzt, aufgrund verschiedener Schriftformen des Baß-Schlüssels, unterschiedliche Zeiten der Einschriften in das Notenbuichlein an. ${ }^{14}$

Für unsere Polonaise Nr. 28, BWV 130, hieße das: Einschrift nicht vor $1733 / 34$.

$\mathrm{Zu}$ dieser Zeit nun müssen Bach und Hasse bereits persönliche Bekanntschaft miteinander geschlossen haben, denn es erscheint einfach undenkbar, daß sich die beiden bekannten Männer 1731 in Dresden bei Bachs Konzerten in der Sophienkirche und anschließend bei Hofe nicht begegnet sein sollen. Hasse weilte bekanntermaßen mit seiner Frau Faustina Bordoni, der weithin in Europa gerühmten Primadonna, in Dresden, um seine Antrittsoper "Cleofide" einzustudieren. $\mathrm{Ob}$ allerdings die besagte CembaloSonate $\mathrm{zu}$ dieser Zeit bereits von Hasse komponiert war, ist eine nicht zu beantwortende Frage. Möglicherweise wurde sie auch durch einen Dresdener Besuch, etwa durch Wilhelm Friedemann, oder durch Silvius Leopold Weiß, vielleicht auch durch Johann Kropffgans, mit nach Leipzig gebracht. Aber dies bleiben, wenn auch glaubhafte, Vermutungen. Die plausibelste Erklärung jedoch sollten wir Forkels Bach-Biographie von 1802 entnehmen: "Bach hatte schon in frühern Jahren dort viele Bekannte, von welchen er allen sehr geehrt wurde. Auch Hasse nebst seiner Gattin,

$"$ Viertel K.-H., Neue Dokumente zu Leben und Werk Johann Adolf Hasses, In: Analecta Musicologica, Band 12, 1973, p. 209-233, Besonders p. 209. Hinsichtlich Hasses und Faustinas Nachlass siehe J. A. Hasse, Ruggiero ovvero l'eroica gratitudine, herausgegeben von Klaus Hortschansky, 1973, Arno Volk Verlag Hans Gerig KG Köln, Concentus Musicus, Veröffentlichungen der musikgeschichtlichen Abteilung des Deutschen Historischen Instituts in Rom, Band I, p. XXIV und VII.

12 Siehe dazu Landmann O., Quellenstudien zum Intermezzo comico per musica und zu seiner Geschichte in Dresden, Phil. Diss, Rostock, masch.

${ }^{13}$ In der Musikbibliothek der Stadt Leipzig befinden sich Hasse-Cembalo-Sonaten in Bearbeitung für Laute (in neufranzösischer Lautentabulatur).

${ }^{14}$ Kritischer Bericht der NBA, p. 71. 
der berühmten Faustina, waren mehrere Mahle in Leipzig gewesen und hatten seine große Kunst bewundert. Er hatte auf diese Weise immer eine ausgezeichnete ehrenvolle Aufnahme in Dresden, und ging oft dahin, um die Oper zu hören. ${ }^{15}$ Sollte nicht der persönliche Kontakt beider, über den allerdings die Quellen beharrlich schweigen, der vernünftigste Grund für das Kennen und die Ubernahme der Polonaise sein? Der frühest-mögliche Termin wäre mit Hasses Dresdener Ankunft (6. Juli 1731) gegeben, theoretisch gegeben, dürfte aber mit Wahrscheinlichkeit doch später liegen. ${ }^{16}$

Das Thema Hasse als Cembalist und Komponist von Musik für das Cembalo ist - trotz des dankenswerten Sonderkapitels in HoffmannErbrechts Studie "Deutsche und italienische Klaviermusik zur Bachzeit" - weithin terra incognita. Wirkt Hasse, wie schon Martin Falck erkannte, ${ }^{17}$ bei seinen Cembalo-Sonaten bereits in die Zukunft, sein Einfluß auf die frühen Werke Carl Philipp Emanuel Bachs wäre hier zu nennen, so vermitteln die leider völlig unbekannten 4 Toccaten Hasses (sie liegen im Archivio Musicale Noseda, Conservatorio "Giuseppe Verdi", Milano) einen stilistisch ganz anderen Hasse, der Bach sehr viel näher steht, als weithin angenommen wird. Hier die Anfangstakte der "Toccata per Cimbalo / del Sigr. Giovanni Adolfo Hasse, detto il Sassone" (Archivio Noseda Milano, Signatur: L 23-22):

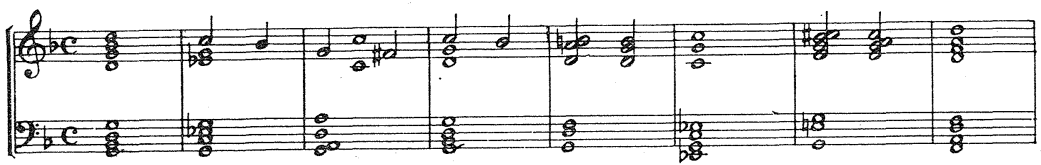

Und nun das Thema der dazugehörigen Fuge:

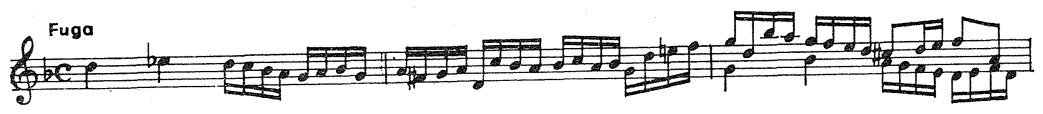

Was kann man nun Konkretes über Hasse, immerhin Schüler Alessandro Scarlattis, als Cembalisten sagen? Daß er in Neapel nicht nur Anerkennung als Komponist errang, sondern er mentzückte auch alle Hörer durch Gesang und Klavierspiel«, berichtet Mennicke, bleibt aber die Quelle dafür leider schuldig. ${ }^{18} \mathrm{Er}$ folgt dabei ganz offenbar dem im umfäglichen HasseArtikel des Musikalischen "Conversations Lexikons" Berichteten von Hermann Mendel, ${ }^{19}$ in welchem Hasse als wein fertiger Klavierspieler" bezeichnet wird. Auf der Kenntnis beider eben angeführter Berichte fußt nun

${ }^{15}$ Forkel J. N., Ueber Johann Sebastian Bachs Leben, Kunst und Kunstwerke. Leipzig, bey Hoffmeister und Kühnel, 1802. Herausgegeben und mit einem Nachwort versehen von Walther Vetter, Henschelverlag Berlin 1968, p. 83-84.

${ }_{16}$ Siehe dazu Hempel G., Bach und Dresden, Jahrbuch zur Pflege der Künste, 5. Folge, Almanach auf das 37. Jahr, Wolfgang Jess Verlag Dresden, p. 45-54.

17 Falck M., Wilhelm Friedemann Bach, C. F. Kahnt/Leipzig 1919, p. 12 und 70.

${ }_{18}$ Mennicke, a. a. O., p. 360.

19 Berlin 1875, Fünfter Band, p. 83. 
offenbar auch Richard Engländers allgemeine Feststellung, im sehr knappen Vorwort zur Veröffentlichung einer Hasseschen F-Dur Cembalo-Sonate (Hoffmann-Erbrecht Nr. 11), daß Hasse ein "sehr guter Pianist" gewesen sei.20 Und damit erschöpft sich die Quellenlage.

Die Frage, wie Hasse dazu kommt Polonaisen zu komponnieren, dürfte weit einfacher $\mathrm{zu}$ beantworten sein. Polnische Musikelemente drangen in der 1. Hälfte des 18. Jahrhunderts, bedingt durch die Tatsache, daß die sächsischen Kurfürsten von 1697 bis 1763 auch Könige Polens waren, zwangsläufig mehr und mehr nach Deutschland.

So tanzte man bei Hof in Dresden u.a. "polnisch". 1709, dieses Datum ist verbürgt, führten Ihro Majestät "mit der Königin unter einer herrlichen Music den Ball ein, dabey pohlnisch getantzt wurde und Paar und Paar Dames und Cavaliers dem König nachfolgten «. ${ }^{21}$ Man sang im polnischen "Gusto" - wie eine Vielzahl von Melodien aus der "Singenden Muse an der Pleiße« beweist, um nur eine jener damals bekannten und äußerst beliebten Liedersammlungen anzuführen.22

Hasse selbst äußerte gegenüber Burney "...die pohlnische Musik sey wirklich national und oft sehr zärtlich und delikat» und schrieb selbst in seiner Germanen-Oper "Arminio» innerhalb der Einleitungs-Sinfonia einen Satz "Alla Polacca".23 Auch in dem Dramma per musica "Zenobia" greift Hasse Anregungen aus der polnischen Musik auf, wenn er im II. Akt eine Arie mit dem Hinweis versieht "nell gusto Polonese«. ${ }^{24}$

Gerade Mitteldeutschland, insbesondere Leipzig, bildete einen Anziehungspunkt für viele Polen, bedingt durch die damals schon wichtige Handels-Messe und durch die Universitäten Leipzig, Halle und Wittenberg, an denen nachweisbar viele Studenten polnischer Herkunft immatrikuliert waren.

Daß sich unter diesen Umständen Polonica in den Werken deutscher Komponisten immer mehr und intensiver niederschlugen, fast Mode wurden, haben gerade in jüngster Zeit einige musikwissenschaftliche Untersuchungen aufgezeigt. 25 In diesem Zusammenhang ist es durchaus nicht

\footnotetext{
${ }^{20}$ bei Kistner und Siegel, Leipzig 1930.

${ }^{21}$ Fassmann D., Das glorwïrdigste Leben und Thaten Friedrich Augusti des Grossen, 1773.

${ }_{22}$ Im Faksimile herausgegeben von Horst Irrgang im VEB Deutscher Verlag für Musik, Leipzig, 1964.

${ }^{23}$ In: Das Erbe Deutscher Musik, Band 27, 1927, Verlag B. Schotts Söhne in Mainz, herausgegeben von Rudolf Gerber.

${ }^{24}$ Chodkowski A., Repertuar muzyczny teatru saskiego $w$ Warszawie, in: Studia Staropolskie, Tom XXXV, Polska Akademia Nauk, Opera w dawnej Polsce na dworze Władysława IV i królow saskich, 1973, p. 167.

${ }^{25}$ Beim Musikwissenschaftlichen Colloquium in Bydgoszcz 1974, siehe Anm. 8, insbesondere die Beiträge von Karol Hławiczka und Zofia Stęszewska, des weiteren: Koch, K.-P., Die Konkordanzen »choreae polonicae» in den Universitätstädten Wittenberg und Leipzig, Kessler F., Die Instrumentalmusik in Gdansk im 16. 18. Jahrhundert, Witkowski L., Zu den Problemen polnisch-deutscher Kontakte in der Hymnologie, dem Studentengesang und der Gesangbewegung, Fleischhauer G., Einflïsse polnischer Musik auf das Schaffen von G. Ph. Telemann, Allihn I., Der Einfluss der polnischen Volksmusik auf das Schaffen und die musikästhe. tische Meinungsbildung Johann Philipp Kirnbergers.
} 
uninteressant festzustellen, daß sich Johann Sebastian Bach dem Einfluß seiner Zeit nicht entzogen hat, wie etwa (um nur ein Beispiel anzuführen) an der "Cantate en burlesque", BWV 212, die gemeinhin als "Bauernkantate» bezeichnet wird, abgelesen werden kann.

\section{POVZETEK}

$\mathrm{V}$ zadnjih letih je bilo ugotovljeno avtorstvo nekaterih del, ki so jih priplsovali J. S. Bachu oziroma glede katerih je bilo dvomljivo, da jih je zložil on. Tako je tudi pisec gornje razprave odkril, da je avtor poloneze št. 28 (G-dur), BWV, dodatek 130, vpisane v klavirsko knjižico za Anno Magdaleno Bach, Johann Adolf Hasse, ki je v letih 1733 do 1763 deloval v Dresdenu kot dvorni kapelnik. Berlinska Državna biblioteka (Staatsbibliothek Preussischer Kulturbesitz) hrani rokopis (BB Mus. ms. 9640), obsegajoč šest Hassejevih simfonij in sedem sonat za cembalo, izdelek neznanega kopista. Druga od omenjenih sonat se zaključuje z dvema polonezama, od katerih je zadnja, ceprav v F-duru, identična $\mathbf{S}$ citirano iz klavirske knjižnice. Obe polonezi kažeta isti potek melodije. Razlike so malenkostne in ne zahtevajo upoštevanja, saj so pri kopistih 18. stoletja pogoste. Vsekakor zanimivejše je vprašanje, ali gre za originalno Hassejevo kompozicijo ali pa le za prevzem oziroma kontrafakturo iz poljske folklore. Z. Stęszewska in K. Hławiczka, izvrstna poznavalca poljske folklore, nista mogla ugotoviti ustreznega vzora originalne poloneze in tako se zdi zelo upravičena domneva, da gre za izvirno delo J. A. Hasseja. Razen tega nas se zanima, kje in kdaj se je Bach seznanil z njegovo polonezo. G. von Dadelsen trdi, da Bach poloneze št. 28, BWV $130 \mathrm{nl}$ zapisal pred letom 1733/34. Bržkone pa sta se skladatelja dotlej že spoznala $v$ Dresdenu. Žal ne vemo, ali je Hasse že tudi do tedaj napisal omenjeno sonato, Dosti enostavnejše je seveda pojasniti vprašanje, kako je Hasse prišel do tega, da je komponiral poloneze. Ker so bili v letih 1687 do 1763 saški volilni knezi tudi poljski kralji, so v prvi polovici 18. stoletja poljski glasbeni elementi vedno bolj prodirali $\mathrm{v}$ Nemčijo in tako se je na dresdenskem dvoru med drugim plesalo tudi po poljsko. 